\title{
Neuroendocrine carcinoma of the ampulla of Vater causing ectopic adrenocorticotropic hormone-dependent Cushing's syndrome
}

\author{
AKIHISA KATO $^{1}$, KAZUKI HAYASHI ${ }^{1}$, ITARU NAITOH ${ }^{1}$, KYOJI SENO $^{2}$, \\ YUKIKO OKADA $^{3}$, TESSHIN BAN ${ }^{1}$, HIROMU KONDO ${ }^{1}$, YUJI NISHI ${ }^{1}$, \\ SHUICHIRO UMEMURA ${ }^{1}$, YASUKI HORI ${ }^{1}$, MAKOTO NATSUME $^{1}$ and TAKASHI JOH ${ }^{1}$ \\ ${ }^{1}$ Department of Gastroenterology and Metabolism, Nagoya City University Graduate School of Medical Sciences, \\ Nagoya 467-8601; ${ }^{2}$ Department of Gastroenterology, Nagoya West Medical Center, Nagoya 462-8508; \\ ${ }^{3}$ Department of Internal Medicine, Kasugai Municipal Hospital, Kasugai 486-8510, Japan
}

Received January 19, 2016; Accepted April 15, 2016

DOI: $10.3892 / \mathrm{mco} .2016 .869$

\begin{abstract}
Ectopic adrenocorticotropic hormone (ACTH) is rarely secreted by neuroendocrine tumors. Although neuroendocrine tumors may occur at any site in the gastrointestinal system, they very rarely occur in the ampulla of Vater and have a poor prognosis. The present study described the first Cushing's syndrome as a result of ectopic ACTH arising from the ampulla of Vater neuroendocrine carcinoma. A 69-year-old female was admitted with clinical features of Cushing's syndrome, confirmed biochemically by hypokalemia, and elevated levels of ACTH and cortisol. In further investigations, a tumor of the ampulla of Vater and liver metastases were detected. Pathological analysis of the biopsy confirmed a neuroendocrine carcinoma, which was immunohistochemically positive for chromogranin A, synaptophysin, cluster of differentiation 56 and ACTH. Therefore, the present study diagnosed a functional and metastatic neuroendocrine carcinoma of the ampulla of Vater with ectopic ACTH production causing Cushing's syndrome. The patient succumbed to mortality 4 months later, despite administration of combined chemotherapy with irinotecan and cisplatin.
\end{abstract}

\section{Introduction}

A neuroendocrine tumor is defined as an epithelial neoplasm that exhibits neuroendocrine differentiation when analyzed by conventional histological, immunohistochemical, ultrastructural and biological evaluations (1). Neuroendocrine tumors of the ampulla of Vater are extremely rare, accounting for $<2 \%$ of

Correspondence to: Dr Kazuki Hayashi, Department of Gastroenterology and Metabolism, Nagoya City University Graduate School of Medical Sciences, 1-Kawasumi, Mizuho-cho, Mizuho-ku, Nagoya 467-8601, Japan

E-mail: khayashi@med.nagoya-cu.ac.jp

Key words: neuroendocrine tumor, ampulla, vater, cushing's syndrome, adrenocorticotropic hormone all ampullary malignancies (2). Therefore, the natural history of this disease entity remains to be established, and its clinical behavior is unknown. Cushing's syndrome, secondary to ectopic adrenocorticotropic hormone (ACTH) secretion, was first described in 1928 and subsequently reported in association with various neuroendocrine tumors (3). The most common primary site of these tumors is the lung, followed by the thymus, pancreas and thyroid (4). Cushing's syndrome arising from the ampulla of Vater has not, to the best of our knowledge, been reported previously. The present study reported the first case of ectopic ACTH secretion from a neuroendocrine tumor of the ampulla of Vater. Written informed consent was obtained from the patient's family.

\section{Case report}

A 69-year-old female presented with general fatigue and edema of the face and legs lasting for 2 months. The patient had not taken any hormones or drugs, nor had she undergone surgery. Physical examination revealed moon face and leg edema. Other physical examination parameters were normal, including blood pressure. Laboratory parameters revealed hypokalemia, with a serum potassium level of $2.5 \mathrm{mmol} / \mathrm{l}$, and slight hyperglycemia, with a fasting blood glucose level of $154 \mathrm{mg} / \mathrm{dl}$. The level of HbAlc was elevated to $6.5 \%$ (normal range, $<6 \%$ ). Further investigations revealed hypercortisolemia and high levels of ACTH and cortisol $(98.2 \mu \mathrm{g} / \mathrm{dl}$ and $567.1 \mathrm{pg} / \mathrm{ml}$, respectively). Plasma cortisol levels at 8 a.m. following overnight 1 and $8 \mathrm{mg}$ dexamethasone suppression tests were 100.4 and $111.0 \mu \mathrm{g} / \mathrm{dl}$, respectively. In view of the high ACTH levels in conjunction with elevated cortisol levels that failed to be suppressed by dexamethasone, the present study diagnosed ectopic ACTH-producing disease. Tumor marker determination revealed increased levels of carcinoembryonic antigen, carbohydrate antigen 19-9 and neuronal-specific enolase (Table I).

The ultrasonography findings were consistent with multiple liver tumors. Contrast-enhanced computed tomography (CT) revealed periampullary growth $(15 \mathrm{~mm})$, dilated common bile duct and main pancreatic duct, masses in both lobes of the liver and hyperplasia of the adrenal glands (Fig. 1A and B). 
Magnetic resonance cholangiopancreatography revealed a soft tissue signal shadow located in the ampulla region, associated with dilation of both the common bile duct and main pancreatic duct. An ${ }^{18} \mathrm{~F}$-flurodeoxyglucose positron emission tomography-CT scan demonstrated an intense uptake of the radiotracer corresponding to the ampulla of Vater, multiple liver deposits and both adrenal glands, however not in any other sites. Head CT revealed no tumor.

The upper gastrointestinal endoscopy revealed a macroscopic ulceration-like lesion at the duodenal papilla (Fig. 1C). Biopsies were obtained from the ampullary mass, which was histologically confirmed as a poorly differentiated neuroendocrine tumor. Immunohistochemical staining was positive for chromogranin A, synaptophysin and cluster of differentiation (CD)56 (Fig. 2). The mitotic rate was 24/10 high-power fields (HPFs) and the Ki-67 index was $80 \%$. A neuroendocrine tumor G3 or neuroendocrine carcinoma was diagnosed, according to the World Health Organization classification. In addition, immunohistochemical staining for ACTH was positive, indicating that the tumor produced ACTH. Therefore, the present study finally diagnosed a functional and metastatic neuroendocrine carcinoma of the ampulla of Vater with ectopic ACTH production causing Cushing's syndrome. Chemotherapy with irinotecan $\left(60 \mathrm{mg} / \mathrm{m}^{2}\right.$, days 1,8 and 15) and cisplatin $\left(60 \mathrm{mg} / \mathrm{m}^{2}\right.$, day 1$)$ on a 28 -day cycle were administered, and also administered mitotane ( $3 \mathrm{~g} /$ day) pre-chemotherapy to control the hypercortisolemic state. However, the hematotoxicity worsened to grade 4 after day 1 administration of the first cycle, and therefore, chemotherapy had to be discontinued. Nevertheless, 1 month later, the patient had a partial response and exhibited improvement on imaging findings and reduced levels of ACTH, cortisol and tumor markers. However, the tumor subsequently regrew and the patient succumbed to liver failure caused by multiple hepatic metastases 4 months after the diagnosis.

\section{Discussion}

Although neuroendocrine tumors, initially assessed as carcinoid tumors, were first described $>100$ years ago, these tumors still raise many issues regarding their classification, prognosis and optimum therapeutic approach (5). According to the World Health Organization classification in 2010, neuroendocrine neoplasms in the digestive system are categorized as neuroendocrine tumors (NET) G1 (carcinoid, mitotic count of $<2 / 10 \mathrm{HPF}$ and/or Ki67 index $\leq 2 \%$ ), NET G2 (mitotic count of 2-20/10 HPF and/or Ki67 index of 3-20\%) and NET G3 (neuroendocrine carcinoma, mitotic count of $>20 / 10$ HPF and/or Ki67 index >20\%) (6). Neuroendocrine tumors rarely arise at the ampulla of Vater, accounting for only $0.05 \%$ of all neuroendocrine tumor types. Its clinical presentation consists of jaundice $(53.1 \%)$, abdominal pain $(24.6 \%)$, pancreatitis $(6.0 \%)$ and weight loss (3.6\%) (7). Almost all neuroendocrine tumors of the ampulla are non-functional tumors and $<3 \%$ of patients with these tumors have hormonal hypersecretion syndrome (8). However, no report of ectopic ACTH secretion associated with neuroendocrine carcinoma of the ampulla of Vater has been published.

Cushing's syndrome, due to ectopic ACTH secretion, occurs in around 5-10\% of all cases of ACTH-dependent
Table I. Laboratory data.

\begin{tabular}{lcc}
\hline Variable & Value & Reference range \\
\hline Potassium & 2.5 & $3.5-4.5 \mathrm{mmol} / \mathrm{l}$ \\
Blood glucose & 154 & $70-109 \mathrm{mg} / \mathrm{dl}$ \\
HbA1c & 6.5 & $<6.0 \%$ \\
Plasma cortisol & 98.2 & $4.5-21.1 \mu \mathrm{g} / \mathrm{dl}$ \\
ACTH & 567.1 & $7.4-55.7 \mathrm{pg} / \mathrm{ml}$ \\
Plasma cortisol after & & \\
1 mg DEX test & 100.4 & $\leq 3.0 \mu \mathrm{g} / \mathrm{dl}$ \\
Plasma cortisol after & & \\
8 mg DEX test & 111.0 & $\leq 1.0 \mu \mathrm{g} / \mathrm{dl}$ \\
CEA & 12.1 & $<5.0 \mathrm{ng} / \mathrm{ml}$ \\
CA19-9 & 101.9 & $<37.0 \mathrm{U} / \mathrm{ml}$ \\
NSE & 27.8 & $<10.0 \mathrm{ng} / \mathrm{ml}$ \\
\hline
\end{tabular}

ACTH, adrenocorticotropic hormone; DEX, dexamethasone; CEA, carcinoembryonic antigen; CA19-9, carbohydrate antigen 19-9; NSE, neuronal-specific enolase.

hypercortisolism (9). These inappropriately high levels of ACTH are secreted by various types of tumor, including neuroendocrine tumors, islet cell tumors, small cell lung carcinomas and medullary thyroid cancers. Clinical features of ectopic ACTH syndrome depend on the source of production and rate of ACTH synthesis. Well-defined changes in Cushingoid body habitus are noticed in slow-growing tumors, whereas rapidly growing tumors, including that observed in the present patient, typically produce a profound and sudden onset of symptoms. Hypokalemia occurs in $80 \%$ of reported cases, including the present patient, and several reports showed that it is more severe compared with that in Cushing's disease (10). The more intensive hypokalemia in ectopic ACTH syndrome can be explained by the mineralocorticoid effect of cortisol, the level of which tends to be higher in this syndrome compared with in Cushing's disease (11).

The mortality and morbidity associated with Cushing's syndrome is associated with the production of excess cortisol. Hypercortisolism results in multiple medical problems, including hypertension, obesity, osteoporosis, fractures, impaired wound healing, infectious diseases, glucose intolerance and psychosis. A previous study indicated that lowering cortisol levels prior to attempting curative treatments (surgery or chemotherapy) may reduce the mortality and morbidity associated with Cushing's syndrome and, in particular, reduce the rates of opportunistic infections (12). Therefore, adrenal blocking agents, including metyrapone or mitotane, may be used to treat hypercortisolemia. In certain patients with hormonal hypersecretion, extensive debulking surgery must be considered even in the presence of distant metastasis (13). However, the present patient had multiple large liver metastatic deposits and therefore, surgery was not deemed an option.

The treatment for neuroendocrine tumors of the ampulla of Vater remains controversial since they are rare tumors with unpredictable biological behavior and prognosis (13). Pancreaticoduodenectomy or local excision has been used 

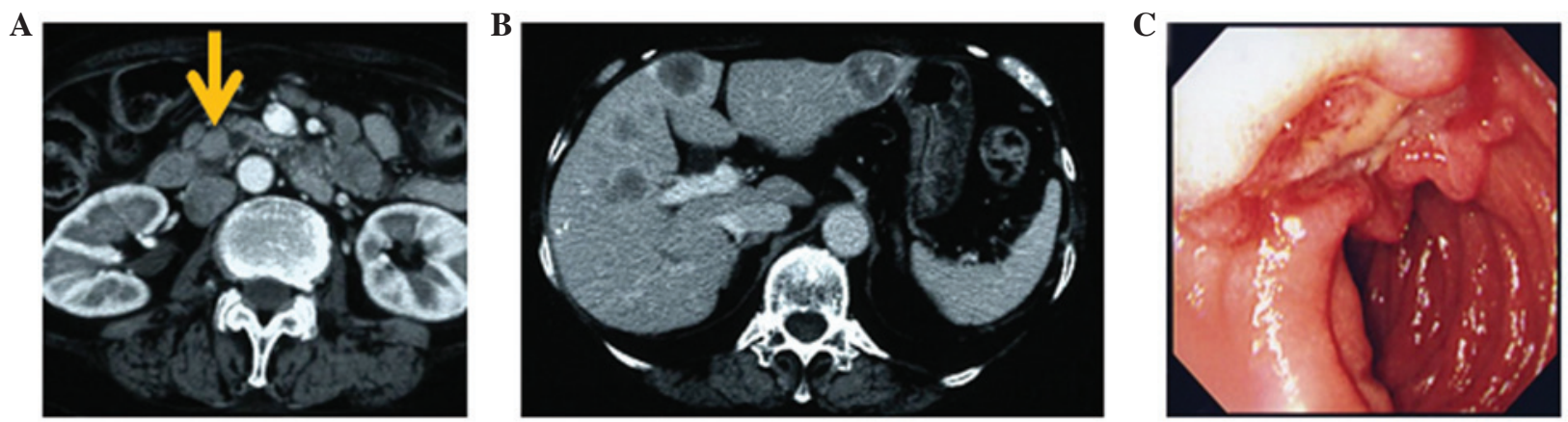

Figure 1. Imaging findings. Abdominal computed tomography findings of the patient indicated (A) a mass in the ampulla of Vater (yellow arrow) and (B) liver metastases. (C) Upper gastrointestinal endoscopy revealed an ulceration-like lesion at the ampulla of Vater.
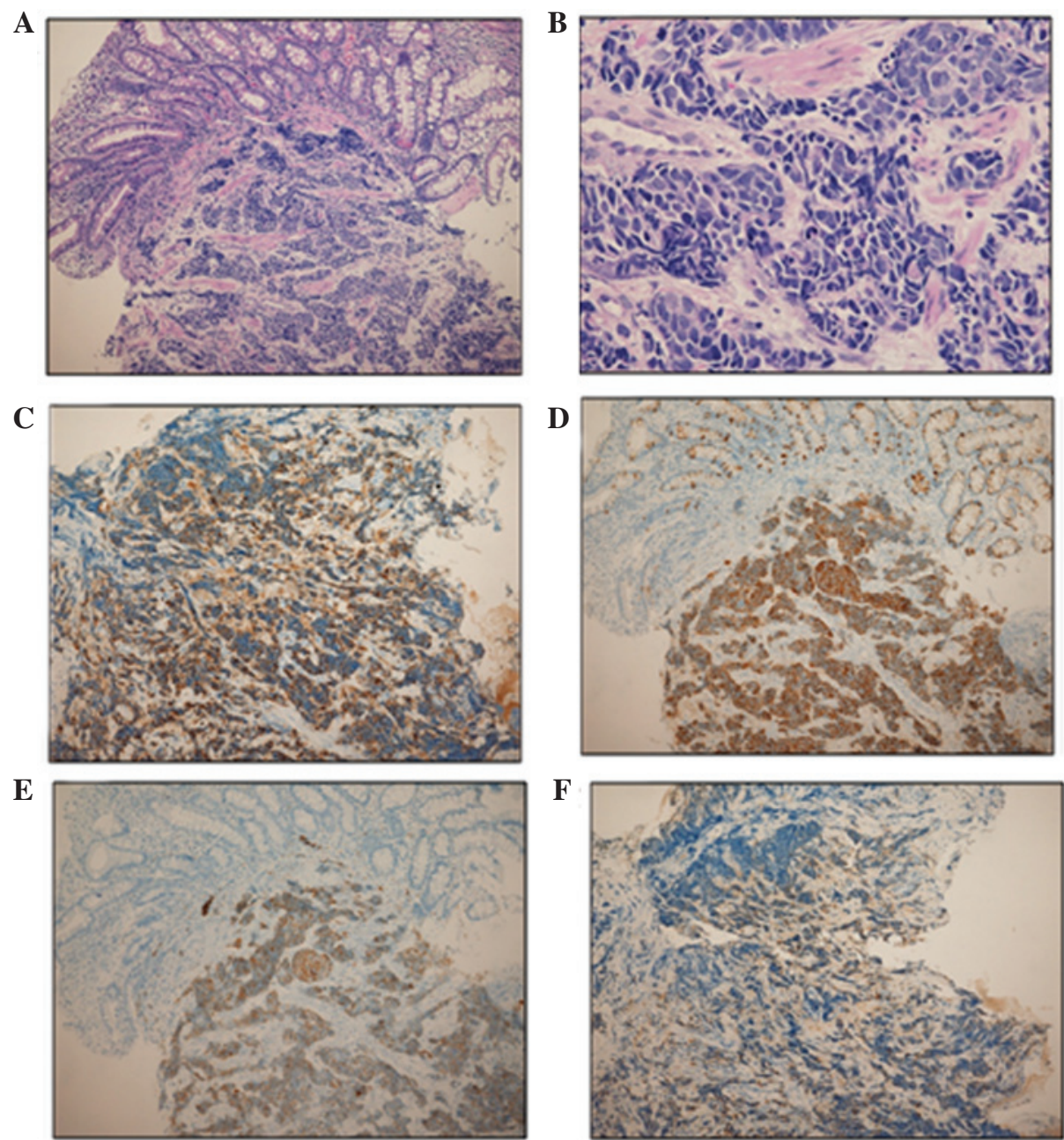

Figure 2. Histological findings. Small atypical cells with nuclear hyperchromatism multiplied in nests and cords were observed by hematoxylin and eosin staining at a magnification of (A) x100 and (B) x400. Immunohistochemical staining revealed that the neuroendocrine tumor cells were positive for (C) chromogranin A, (D) synaptophysin, (E) cluster of differentiation 56 and (F) adrenocorticotropic hormone (magnification, x100).

for these tumors of all sizes with no distant spread $(14,15)$. Although generally more indolent compared with carcinomas, once they progress beyond surgical resectability, they are essentially incurable. Therefore, systemic treatment options for the management of advanced disease have expanded substantially in recent years. Not only distant metastasis, but also tumor grade are important prognostic factors for survival in patients with neuroendocrine tumors of the ampulla of Vater (14). Low-grade tumors show 5- and 10-year survival rates of 80 and $71 \%$, respectively, whereas high-grade neuroendocrine tumors have dismal 5- and 10-year survival rates (15\%) (7). Due to the paucity of reported cases of neuroendocrine carcinoma, no standardized chemotherapy exists for this tumor type. Chemotherapeutic regimens, including cisplatin, irinotecan, etoposide, doxorubicin and vincristine have been reported. Large-scale retrospective analyses of advanced gastrointestinal and pancreatic neuroendocrine carcinoma by a Japanese group demonstrated that irinotecan plus cisplatin 
and etoposide plus cisplatin are the most commonly used regimens (16).

In conclusion, this is the first report, to the best of our knowledge, describing neuroendocrine carcinoma of the ampulla of Vater responsible for ectopic production and secretion of ACTH and resulting in Cushing's syndrome. Further data and therapies, particularly effective adjuvant chemotherapies, are urgently required to improve the survival rates of patients with neuroendocrine tumors of the ampulla of Vater, a rare but aggressive tumor.

\section{References}

1. Selvakumar E, Rajendran S, Balachandar TG, Kannan DG, Jeswanth S, Ravichandran P and Surendran R: Neuroendocrine carcinoma of the ampulla of Vater: A clinicopathologic evaluation. Hepatobiliary Pancreat Dis Int 7: 422-425, 2008.

2. Ricci JL: Carcinoid of the ampulla of Vater. Local resection or pancreaticoduodenectomy. Cancer 71: 686-690, 1993.

3. Riggs BL Jr and Sprague RG: Association of Cushing's syndrome and neoplastic disease: Observations in 232 cases of Cushing's syndrome and review of the literature. Arch Intern Med 108: 841-849, 1961.

4. Jex RK, van Heerden JA, Carpenter PC and Grant CS: Ectopic ACTH syndrome. Diagnostic and therapeutic aspects. Am J Surg 149: 276-282, 1985.

5. Oberg K: Neuroendocrine tumors (NETs): Historical overview and epidemiology. Tumori 96: 797-801, 2010.

6. Rindi G, Petrone G and Inzani F: The 2010 WHO classification of digestive neuroendocrine neoplasms: A critical appraisal four years after its introduction. Endocr Pathol 25: 186-192, 2014.

7. Albores-Saavedra J, Hart A, Chablé-Montero F and Henson DE: Carcinoids and high-grade neuroendocrine carcinomas of the ampulla of vater: A comparative analysis of 139 cases from the surveillance, epidemiology, and end results program-a population based study. Arch Pathol Lab Med 134: 1692-1696, 2010.
8. Solcia E, Fiocca R, Rindi G, Villani L, Luinetti O, Burrell M, Bosi F and Silini E: Endocrine tumors of the small and large intestine. Pathol Res Pract 191: 366-372, 1995.

9. Wajchenberg BL, Mendonca BB, Liberman B, Pereira MA Carneiro PC, Wakamatsu A and Kirschner MA: Ectopic adrenocorticotropic hormone syndrome. Endocr Rev 15: 752-787, 1994.

10. Stewart PM, Walker BR, Holder G, O'Halloran D and Shackleton CH: 11 beta-Hydroxysteroid dehydrogenase activity in Cushing's syndrome: Explaining the mineralocorticoid excess state of the ectopic adrenocorticotropin syndrome. J Clin Endocrinol Metab 80: 3617-3620, 1995.

11. Arteaga E, Fardella C, Campusano C, Cárdenas I and Martinez P. Persistent hypokalemia after successful adrenalectomy in a patient with Cushing's syndrome due to ectopic ACTH secretion: Possible role of 11beta-hydroxysteroid dehydrogenase inhibition. J Endocrinol Invest 22: 857-859, 1999.

12. Ejaz S, Vassilopoulou-Sellin R, Busaidy NL, Hu MI, Waguespack SG, Jimenez C, Ying AK, Cabanillas M, Abbara M and Habra MA: Cushing syndrome secondary to ectopic adrenocorticotropic hormone secretion: The university of texas MD anderson cancer center experience. Cancer 117: 4381-4389, 2011.

13. Klimstra DS, Modlin IR, Coppola D, Lloyd RV and Suster S: The pathologic classification of neuroendocrine tumors: A review of nomenclature, grading, and staging systems. Pancreas 39: 707-712, 2010.

14. Jayant M, Punia R, Kaushik R, Sharma R, Sachdev A, Nadkarni NK and Attri A: Neuroendocrine tumors of the ampulla of vater: Presentation, pathology and prognosis. JOP 13: 263-267, 2012

15. Odabasi M, Yildiz KM, Cengiz E, Hasan AH, Gunay E, Ozkan E, Aktekin A, Kaya B and Muftuoglu TM: Treatment of ampullary neuroendocrine tumor by endoscopic snare papillectomy. Am J Case Rep 14: 439-443, 2013.

16. Yamaguchi T, Machida N, Morizane C, Kasuga A, Takahashi H, Sudo K, Nishina T, Tobimatsu K, Ishido K, Furuse J, et al: Multicenter retrospective analysis of systemic chemotherapy for advanced neuroendocrine carcinoma of the digestive system. Cancer Sci 105: 1176-1181, 2014. 